\title{
Spliced leader trans-splicing in the nematode Trichinella spiralis uses highly polymorphic, noncanonical spliced leaders
}

\author{
JONATHAN PETTITT, BERNDT MÜLLER, IAN STANSFIELD, and BERNADETTE CONNOLLY \\ School of Medical Sciences, Institute of Medical Sciences, University of Aberdeen, Aberdeen AB25 2ZD, United Kingdom
}

\begin{abstract}
The trans-splicing of short spliced leader (SL) RNAs onto the $5^{\prime}$ ends of mRNAs occurs in a diverse range of taxa. In nematodes, all species so far characterized utilize a characteristic, conserved spliced leader, SL1, as well as variants that are employed in the resolution of operons. Here we report the identification of spliced leader trans-splicing in the basal nematode Trichinella spiralis, and show that this nematode does not possess a canonical SL1, but rather has at least 15 distinct spliced leaders, encoded by at least 19 SL RNA genes. The individual spliced leaders vary in both size and primary sequence, showing a much higher degree of diversity compared to other known trans-spliced leaders. In a survey of $T$. spiralis mRNAs, individual mRNAs were found to be trans-spliced to a number of different spliced leader sequences. These data provide the first indication that the last common ancestor of the phylum Nematoda utilized spliced leader trans-splicing and that the canonical spliced leader, SL1, found in Caenorhabditis elegans, evolved after the divergence of the major nematode clades. This discovery sheds important light on the nature and evolution of mRNA processing in the Nematoda.
\end{abstract}

Keywords: nematode; Trichinella; spliced leader; trans-splicing

\section{INTRODUCTION}

Trans-splicing involves the intermolecular ligation of exons derived from two independent RNA molecules. The transsplicing of short RNA-leader, or spliced leader (SL), sequences onto the $5^{\prime}$ ends of pre-mRNAs is found in a variety of eukaryotic taxa, including protists (Sutton and Boothroyd 1986; Tessier et al. 1991; Zhang et al. 2007), cnidarians (Stover and Steele 2001), platyhelminths (Davis 1997; Zayas et al. 2005), nematodes (Krause and Hirsh 1987; Spieth et al. 1993; Guiliano and Blaxter 2006), rotifers (Pouchkina-Stantcheva and Tunnacliffe 2005), and tunicates (Vandenberghe et al. 2001; Ganot et al. 2004; Satou et al. 2006). These spliced leaders are donated by longer, precursor SL RNAs through a splicing reaction that is mechanistically similar to the cis-splicing used to remove

Reprint requests to: Jonathan Pettitt, School of Medical Sciences, Institute of Medical Sciences, University of Aberdeen, Aberdeen AB25 2ZD, United Kingdom; e-mail: j.pettitt@abdn.ac.uk; or Bernadette Connolly, School of Medical Sciences, Institute of Medical Sciences, University of Aberdeen, Aberdeen AB25 2ZD, United Kingdom; e-mail: b.connolly@ abdn.ac.uk; fax: 44-1224-555844.

Article published online ahead of print. Article and publication date are at http://www.rnajournal.org/cgi/doi/10.1261/rna.948008. introns from precursor mRNAs. The primary sequences of the respective SL RNAs are not conserved between these groups, but share common structural features. In all cases the SL is located at the $5^{\prime}$ end of the SL RNA and is followed by an intron that is removed during the ligation of the SL onto the splice acceptor site present on the transspliced pre-mRNA. All SL RNAs form a stem-loop structure such that the donor splice site is base-paired to the SL sequence. This structure is analogous to the base pairing between U1 snRNA and the donor splice site in cis-splicing. The intron also contains a binding site for Sm proteins, and in many SL RNAs this site is flanked by stem-loop structures. The conservation of these features has led to the proposal that trans-splicing is an ancestral eukaryotic trait; however the alternative explanation, that SL trans-splicing has arisen independently multiple times, cannot be excluded (Nilsen 2001; Hastings 2005).

In nematodes, spliced leader trans-splicing was first described in Caenorhabditis elegans (Krause and Hirsh 1987), whereby a common 22-nucleotide (nt) RNA molecule (SL1) is donated by a 100-nt SL RNA onto the 5' ends of $\sim 70 \%$ of mRNAs. A related spliced leader, SL2, is added to the mRNAs derived from genes that lie downstream of the first gene in operons, as part of the processing of the 
polycistronic RNAs produced by such gene clusters (Spieth et al. 1993). C. elegans has 110 copies of the SL1 RNA gene, arranged in tandem with the 5S rRNA gene. In contrast, SL2 is encoded by 18 genes that are dispersed throughout the genome. These show a limited degree of sequence variability, but there does not appear to be a bias in their participation in trans-splicing (Blumenthal 2005). SL1 has been identified in all nematodes studied to date, suggesting that it may have arisen in the ancestor of all modern nematodes, whereas SL2 seems likely to have evolved as a variant of SL1, in the ancestor of the Rhabditina (the nematode clade, which includes $C$. elegans). Operons are more widespread than SL2, being found in the more distantly related nematodes of the Tylenchina and Spirurina clades, where polycistronic RNAs produced from operons are resolved by SL1 and SL1-like variants (Guiliano and Blaxter 2006).

An important question is whether spliced leader transsplicing is a common feature of all modern nematodes. The available data on trans-splicing in the phylum Nematoda is derived from nematodes that fall within the same subclass, Chromadoria (Guiliano and Blaxter 2006). Evidence for the existence of trans-splicing in the more basal nematode subclasses of Enoplia and Dorylaimia has been missing or inconclusive (Lindh et al. 1998). Serendipitous observations of discrepancies in the comparison between $5^{\prime}$ rapid amplification of cDNA ends ( $5^{\prime} \mathrm{RACE}$ ) cDNA and genomic sequences derived from two genes of the Dorylaimid Trichinella spiralis suggested to us the possibility that these genes were trans-spliced and provided the impetus to investigate this likelihood further. Here we show that $T$. spiralis engages in spliced leader trans-splicing, but that it does not possess SLs that resemble SL1, SL2, or any other nematode SLs. Uniquely, it has at least 19 active SL RNA genes, which are highly polymorphic in their primary sequences, all of which are located within the same region of the genome. The diversity of SL sequences is unprecedented and raises the possibility that the use of a limited number of highly conserved SLs, such as is found in the Chromodoria, does not represent the ancestral state for the nematode phylum. However, our definitive identification of trans-splicing in a Dorylaimid nematode confirms that trans-splicing is an ancestral nematode trait.

\section{RESULTS}

\section{mRNAs in $T$. spiralis are trans-spliced using multiple distinct spliced leaders}

During the characterization of $5^{\prime} \mathrm{RACE}$ cDNA sequences derived from two $T$. spiralis genes, blgs-1 (Bcl9/legless homolog 1) and tll-2 (TGF-beta-like ligand 2) (GenBank accession numbers EF682094 and EF681125, respectively), we noticed that the first 21-22 nt did not derive from genomic DNA found upstream or anywhere within $50 \mathrm{~kb}$ of the two genes (data not shown). A draft Trichinella spiralis genome sequence, which has been produced by the Genome Sequencing Center at Washington University School of Medicine in St. Louis, allowed a comparison of cDNA and genomic sequences. Inspection of the genomic sequence for the two genes revealed that the points at which the genomic and cDNA sequences no longer matched corresponded to consensus splice acceptor sites (Table 1). In addition, no cDNA suggested that there were any exons upstream of the acceptor site for either of the genes. Such "orphan" splice acceptors sites have been shown in $C$. elegans to be predictive of genes whose mRNAs are transspliced to spliced leaders, leading to replacement of the $5^{\prime}$ UTR, or "outron," with the spliced leader (Conrad et al. 1991). Given that the trans-splicing of 22-nt spliced leaders to mRNAs is a common feature found in all characterized nematodes, it seemed likely that these additional nucleotides corresponded to $T$. spiralis spliced leaders. We obtained in total $255^{\prime}$ RACE cDNA sequences for blgs-1 and four for tll-2, all but two of which had putative spliced leader sequences at their $5^{\prime}$ ends (Table 1). This revealed a total of 10 distinct putative spliced leader sequences, none of which showed sequence identity with the canonical nematode spliced leader SL1 (5'-GGTTTAATTACCCAAGTTT GAG-3'), or to any of its derivatives. This survey demonstrated that pre-mRNAs from the same gene can utilize multiple spliced leaders and furthermore revealed instances of the same spliced leaders being found in cDNAs from both blgs-1 and tll-2. Since these genes map to different supercontigs, it is unlikely that the leader sequences are added via long-range cis-splicing.

We named the spliced leaders trans-spliced leaders 1-10 (TSL1-10) to distinguish them from the SL1 and SL2 of other nematodes. To determine whether these spliced leaders were found at the $5^{\prime}$ ends of other mRNAs we carried out sequence similarity searches against the publicly available $T$. spiralis ESTs $(\sim 22,000)$ for cDNAs whose $5^{\prime}$ ends matched all or part of the TSLs (Supplemental Table 1). All matches were screened to confirm that the position of the spliced leader in the cDNA sequence correlated with a consensus $T$. spiralis splice acceptor site (5'TTT(T/C)AG-3') (B. Connolly, unpubl.) in the corresponding genomic sequence. For four of these genes whose mRNAs were identified as SL trans-splicing targets (DQ132801, GM2 activator protein GM2-AP; BG353788, cytoskeleton associated protein CKAP1; BG731798, RNA helicase; BG732141, Bcl-X homolog) we independently confirmed that their mRNAs received spliced leaders by using $5{ }^{\prime}$ RACE (Table 1). This led to the identification of two new TSLs (TSL11, TSL12), bringing the total of distinct spliced leaders being actively used in trans-splicing to 12 . Three additional putative spliced leaders (TSL13-15) were later defined by further computational identification of TSL RNA genes (see below). We also carried out similarity searches of the EST database with SL1 and SL2 but failed to 
TABLE 1. 5' UTR nucleotide sequence of $T$. spiralis trans-spliced cDNA clones

$5^{\prime}$-End sequence of 5'RACE cDNA clones ${ }^{\mathrm{a}}$

TSL

No

blgs- $7^{\mathrm{b}}$

Genomic DNA sequence: TTTTAGCTGCTTGTTTTTCGGAAACAGCTTCTGTGTATG AGGTATTTACCAGATCTAAAAGCTGCTTGTTTTTCGGAAACAGCTTCTGTGTATG AGGTATTTACCAGgTCTAAAAGCTGCTTGTTTTTCGGAAACAGCTTCTGTGTATG AGGTATTTACCAGATtTAAAAGCTGCTTGTTTTTCGGAAACAGCTTCTGTGTATG AGGTATTTACCGAATTAAAAAGCTGCTTGTTTTTCGGAAACAGCTTCTGTGTATG AGGTATTTACCGAATCAAAAAGCTGCTTGTTTTTCGGAAACAGCTTCTGTGTATG cGGTACTTACCGAATTAAAAAGCTGCTTGTTTTTCGGAAACAGCTTCTGTGTATG GGTTATTTACCGAACTTAAAAGCTGCTTGTTTTTCGGAAACAGCTTCTGTGTATG GCGATTGTTCGAATTTACTTGAAGCTGCTTGTTTT-CGGAAACAGCTTCTGTGTATG AAATACCTTTCAATTTGTTTGAAGCTGCTTGTTTTTCGGAAACAGCTTCTGTGTATG AACCTGCACGACTTGTTCGAAGCTGCTTGTTTTTTCGGAAACAGCTTCTGTGTATG AACCTGCACGACTTGTTCGgAGCTGCTTGTTTTTCGGAAACAGCTTCTGTGT ATG ATCTGTCGGTATTCCTGAAAGCTGCTTGTTTTTTCGGAAACAGCTTCTGTGTATG AcACGTGGTTATTTATTGAAGCTGCTTGTTTTTCGGAAACAGCTTCTGTGTATG GGTAATATTTACTGAATTCAAGCTGCTTGTTTTTCGGAAACAGCTTCTGTGTATG AATGTTGCTATACATTTTAGCTGCTTGTTTTTCGGAAACAGCTTCTGTGTATG ATTTTAGCTGCTTGTTTTTCGGAAACAGCTTCTGTGTATG

$t 11-2^{c}$

Genomic DNA sequence: TTTAAGGAAAACCCCTATG AAATACCTTTCAATTTGTTTGAAGGAAAACCCCTATG AACCTTTGCGCATCGTTTAAAGGAAAACCCCTATG AACCTGCACGACTTGTTCGAAGGAAAACCCCTATG ATCTGTCGGTATTCCTGAAAGGAAAACCCCTATG

GM2 activator protein ${ }^{\mathrm{d}}$

Genomic DNA sequence: TTGCAGTGTTGCTGAAAAATG AGGTATTTACCAGATCTAAAAGTGTTGCTGAAAAATG AGGTATTTACCGAATTAAAAAGTGTTGCTGAAAAATG GGTTATTTACCGAACTTAAAAGTGTTGCTGAAAAATG AACCTTTGCGCATCGTTTAAAGTGTTGCTGAAAAATG AACCTGCACGACTTGTTCGAAGTGTTGCTGAAAAATG ATCTGTCGGTATTCCTGAAAGTGTTGCTGAAAAATG AACCTTTGAACCCACTTCAAGTGTTGCTGAAAAATG

Cytoskeleton associated protein ${ }^{\mathrm{e}}$

Genomic DNA sequence: TTTTAGCTAATAAAATG ATCTGTCGGTATTCCTGAAAGCTAATAAAATG AACCTGCACGACTTGTTCGAAGCTAATAAAATG

RNA helicase $\mathrm{f}^{\mathrm{f}}$

Genomic DNA sequence: TTACAGCGTATTCAGAAGAATG CAGACGTGGTTATTTATTGAAGCGTATTCAGAAGAATG AAATCAGTATATTGTGTTTGTACCACCGGAAGTTTTGCTTTGTTTGTAATTCTCTTATTTTACGTATTCAGAAGAATG TATATTGTGTTTGTACCACCGGAAGTTTTGCTTTGTTTGTAATTCTCTTATTTTACGTATTCAGAAGAATG ATATTGTGTTTATATCACCGGAAGTTTTGCTTTGTTTGTAATTCTCTTATTTTTACGTATTCAGAAGAATG TTGT-CACCGGAAGTTTTGCTTTGTTTGTAATTCTCTTATTTTACGTATTCAGAAGAATG

Ts-bcl- $\mathrm{X}_{\mathrm{L}}^{\mathrm{g}}$

Genomic DNA sequence: ITTTAGGTTGTAATTTATATATTCACGATG AACCTTTGCGCATCGTTTAAAGGTTGTAATTTATATATTCACGATG ACGAATTTACCGTATTTGTCAAGGTTGTAATTTATATATTCACGATG AACTTACAGTATGTCGTTGTTCAAACTGTGTTGCTGACTGTCTTTGCTGAATTCTCGGTTGTAATTTATATATTCACGATG GTTGCTGACTGTCTTTGCTGAATTCTCGGTTGTAATTTATATATTCACGATG CTTTTCAAAGTTCCATTTTCTTCGAATGTGTGGTTGTAATTTATATATTCACGATG

(NS) not spliced; (CS) cis-spliced; $(*)$ TSL variants.

aTSL sequence shown in bold; initiator ATG codon of protein coding sequence is shaded; splice acceptor site in genomic DNA is underlined. ${ }^{\mathrm{b}}$ GenBank accession number EF682094.

${ }^{\mathrm{C}}$ GenBank accession number EF681125.

${ }^{\mathrm{d}}$ GenBank accession number DQ132801.

${ }^{\mathrm{e}}$ GenBank accession number BG353788.

${ }^{f} G e n B a n k$ accession number BG731798.

${ }^{\mathrm{g}}$ GenBank accession number BQ732141. 
identify any ESTs that were trans-spliced with either spliced leader sequence. Among the SL trans-spliced ESTs there was a clear preference for utilization of TSL1-4 (Fig. 1A), but whether this reflects a real bias, or a bias in the selection of clones for sequencing and/or library construction remains to be determined. This analysis further showed that TSL addition is not confined to muscle L1 larva, but also occurs in the immature L1 larva and in the adult worm. The relatively few numbers of immature L1 or adult ESTs identified probably reflects the fact that ESTs in the database are predominantly derived from the muscle L1 larva.

The 5'RACE sequence analysis revealed that three of the six genes produced mRNAs that did not always receive a spliced leader, as has been observed in other organisms (Zayas et al. 2005; Satou et al. 2006). Two of the 25 blgs-1 5'RACE clones did not show addition of a spliced leader, instead beginning with sequences derived from the outron (Fig. 1B). The RNA helicase and Bcl-X homolog genes fell into a different category where we recovered products of both trans-splicing and cis-splicing: six of the seven RNA helicase cDNAs were cis-spliced to an upstream noncoding exon whereas only one was trans-spliced (Fig. 1C). This could represent competition between cis- and trans-splicing or could be due to the presence of an alternative promoter
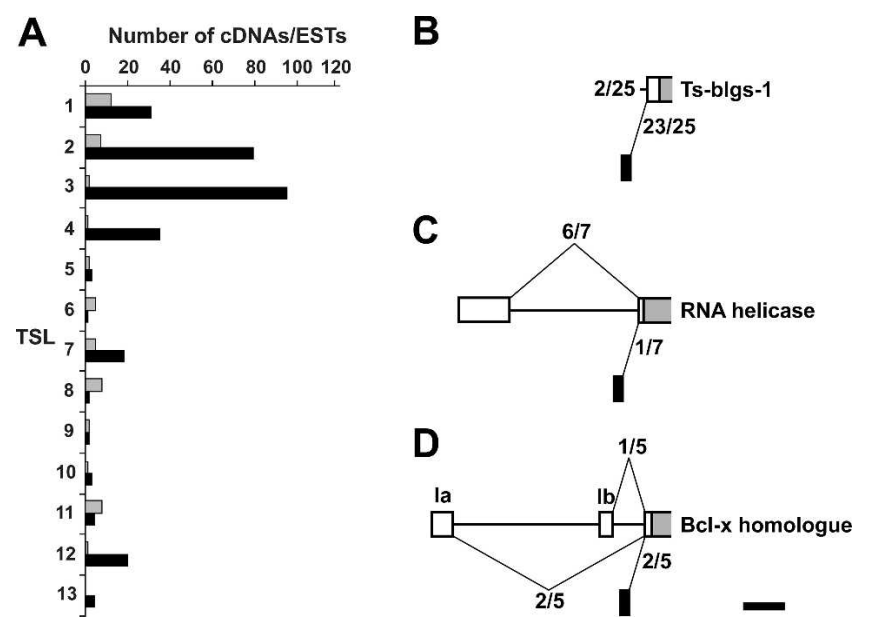

FIGURE 1. Patterns of TSL addition to Trichinella spiralis mRNAs. (A) Graph showing the number of times that each TSL was detected at splice acceptor sites, identified via $5^{\prime}$ RACE (gray bars) or in a survey of publicly available EST sequences $(\sim 22,000)$ (black bars). $(B-D)$ Instances of TSL trans-splicing detected in $5^{\prime}$ RACE analysis of blgs- 1 and genes represented by BG731798 (RNA helicase) and BQ732141 (bcl- $\mathrm{X}_{\mathrm{L}}$ ). (B) An example of a gene that utilizes a classic outron splice acceptor site: a splice site that is involved in spliced leader transsplicing, but not in cis-splicing. (C,D) Examples of trans-splice acceptor sites that can also participate in cis-splicing to upstream donor splice sites. Exons are denoted by boxes, with shading used to indicate open reading frames; unshaded regions denote untranslated regions. Introns and intergenic regions are represented by horizontal lines. The spliced leader is represented by a black box. In each case the number of representative clones as a fraction of the total number analyzed is given. The scale bar indicates $100 \mathrm{nt}$. located in the intronic sequence. This alternative promoter use has been observed for C. elegans genes. The final example is that of the Bcl-X gene, representing a third category in which two of the five $5^{\prime} \mathrm{RACE}$ cDNAs are transspliced and the remaining three are cis-spliced but to two alternative upstream noncoding exons (Fig. 1D). Interestingly, we found that among the seven Bcl-X ESTs available from the public database that included $5^{\prime}$-end cDNA sequence only two are trans-spliced; the remaining five are cis-spliced to an upstream exon (Fig. 1D, Ia). In addition, all of the twenty $5^{\prime} \mathrm{RACE}$ clones we sequenced for the GM2 activator protein were found to be transspliced; this compares well with the 13 full-length GM2activator protein ESTs present in the database, of which 12 are trans-spliced (Supplemental Table 1) and one is not spliced. We also noticed an apparent gene-specific bias in TSL trans-splicing. TSL- 1 is the most frequently added to blgs-1 mRNAs (11/23), whereas for the GM2 activator protein mRNA it is TSL-11 $(8 / 20)$ that is most commonly added. One possible explanation for this observed bias is tissue-specific expression of the respective TSLs.

\section{Identification of $T$. spiralis SL RNA genes}

Taken together the cDNA data suggested that we had identified spliced leader sequences added to the mRNAs by trans-splicing. If these additional sequences represent genuine $T$. spiralis spliced leaders then we expected that we should be able to identify the genes that encode them by searching through the complete $T$. spiralis genome for regions that exactly matched the spliced leader sequence. As predicted, using this strategy we were able to identify matches to all of the putative spliced leaders (Fig. 2A; Table 2). A number of TSLs identified from cDNA sequences showed single nucleotide mismatches when aligned to the T. spiralis genome sequence. These mismatches might have arisen from errors introduced during PCR amplification or could indicate allelic variants. Strikingly, all the matches were confined to supercontig 0 (Fig. 2A), suggesting that as in other organisms the trans-spliced leader RNA ( $t s l r)$ genes in T. spiralis are located together. Notably, and in contrast to a number of other species including $C$. elegans, the tslr genes are not organized in clusters with the 5S rRNA genes, although these latter rRNA genes are also found on supercontig 0 . The $t s r l$ genes are found over an $8-\mathrm{Mb}$ region interspersed with putative protein coding genes. Some of the $t s l r$ genes appear to be single transcription units (e.g., tslr-10, tsrl-8), whereas others appear to be grouped (tslr4.1, tslr-4.2, tsrl-5.1, tslr-5.2, tslr-5.3, tslr-14.1, tslr-14.2), although they are not necessarily transcribed in the same direction.

All known nematode spliced leader RNAs are produced as $~ 100$-nt precursor RNAs consisting of the 22-nt spliced leader followed by a splice donor site used to trans-splice the SL onto the target mRNA. The remainder of the SL 


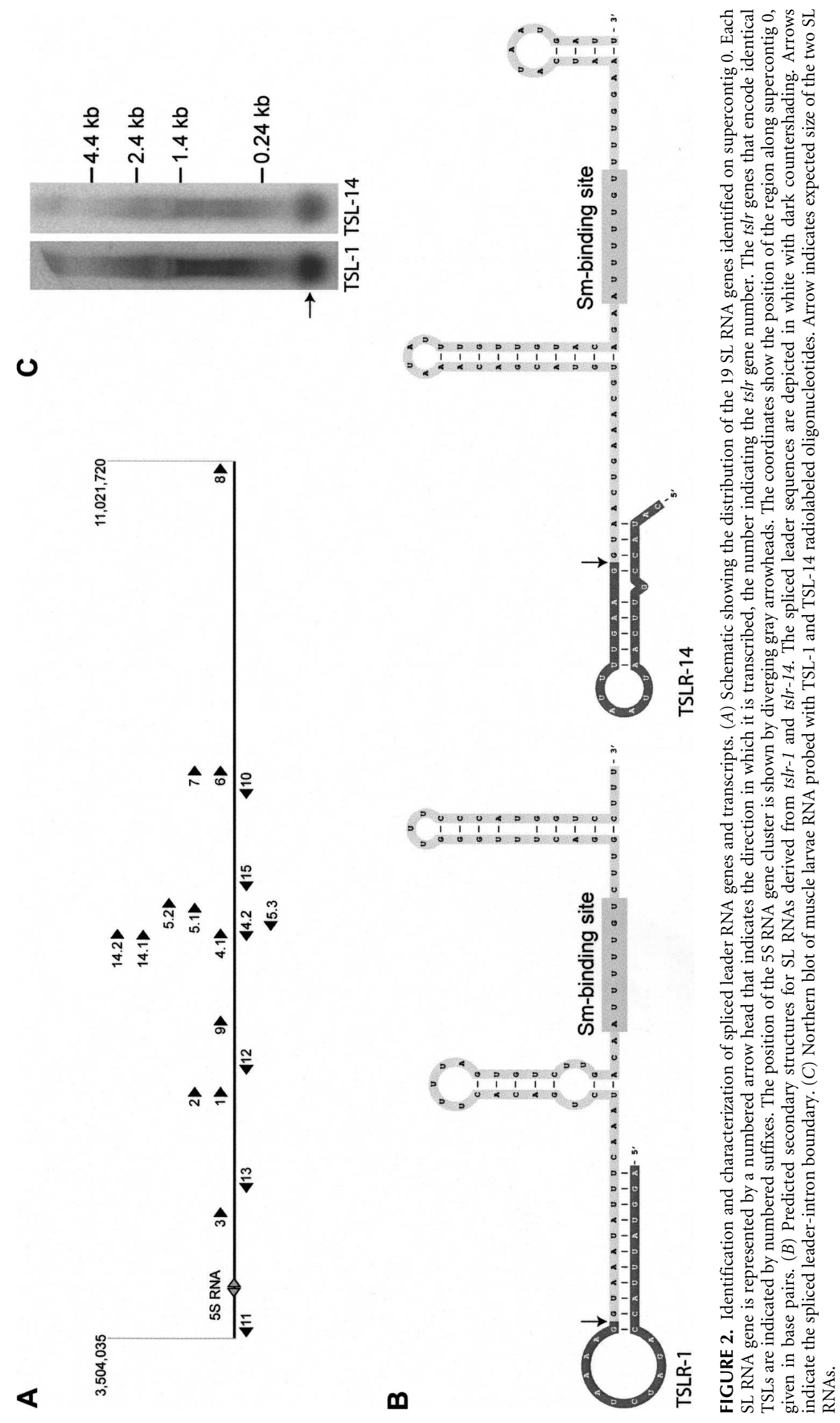




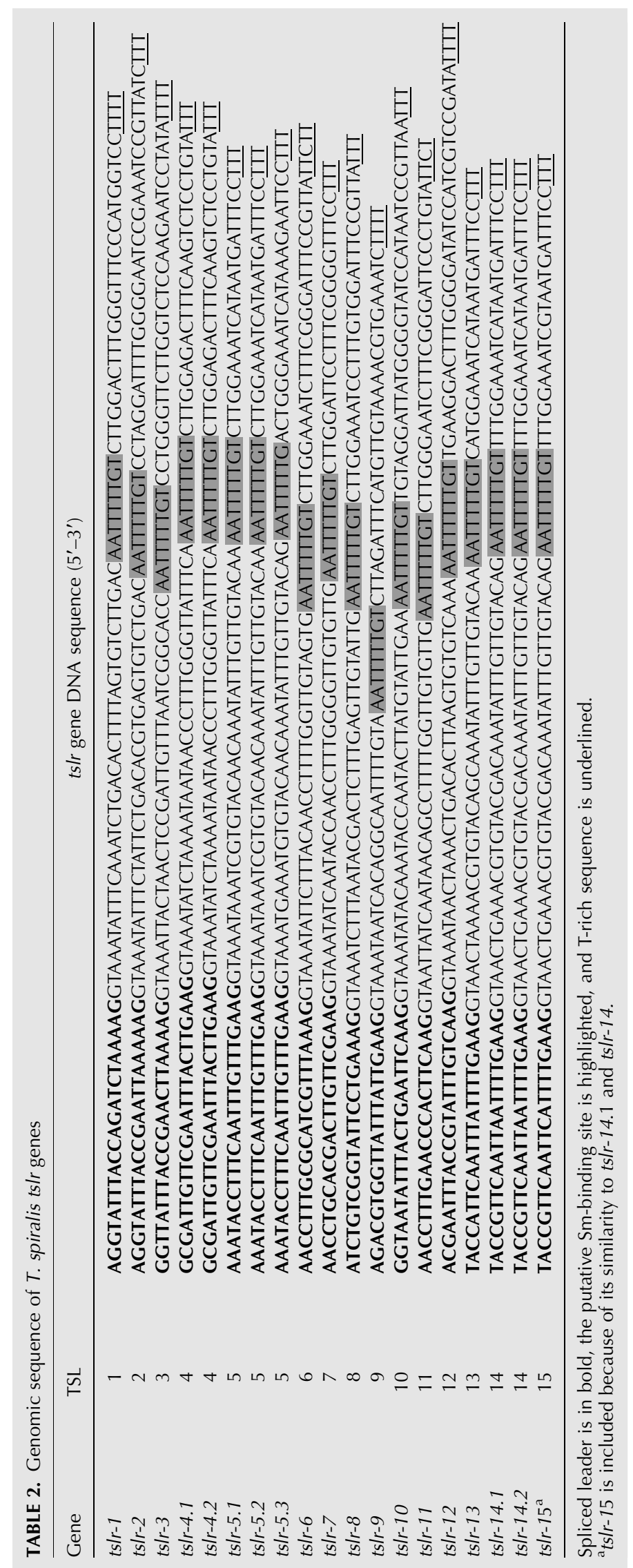


RNA, usually referred to as the SL intron, is removed during trans-splicing. The 100-nt SL RNAs adopt a characteristic secondary structure whereby the spliced leader base pairs with parts of the intron, forming a hairpin. Two additional hairpins in the intron flank the Sm binding site. We thus examined the DNA downstream of the matches to the putative splice leaders for evidence of these structures. We found that all contained the consensus splice donor site $\left(5^{\prime}\right.$-GTAA-3') immediately $3^{\prime}$ of the predicted splice leader that in RNA folding predictions was contained within a hairpin. The spliced leader was followed by two regions that were again predicted to be able to form hairpins, and between them was a stretch of nucleotides ( $5^{\prime}$-ATTTTTGT$\left.3^{\prime}\right)$ that closely matched the consensus Sm binding site in C. elegans (5'-purine-AU ${ }_{4-6} \mathrm{G}$-purine-3') (Mattaj 1988; Thomas et al. 1990).

In order to carry out a more exhaustive search for SL RNA genes we used an algorithm to search for combinations of conserved features diagnostic for the SL RNA genes that we had already identified on the basis of TSL sequence identity. Using this approach we were able to identify an additional 29 predicted $t s l r$ genes (Table 2; Supplemental Table 2), although at present we have no experimental evidence that any of these are actively used. Thus, in total we were able to find potentially 48 individual $t s l r$ genes, 28 of which are located in the cluster on supercontig 0 and the remaining 20 distributed over a number of other supercontigs. Large gaps in the genomic sequence of supercontig 0 still exist, and it is likely that as these are filled in additional $t$ slr genes will be uncovered. Indeed our analysis of the EST database has identified putative orphan TSLs (Supplementary Table 1), not found in the currently available genomic sequence. Furthermore, in our searches we failed to find any evidence for the presence of SL RNA genes encoding the "cannonical" nematode SL1 or SL2 or any of the known variants (Guiliano and Blaxter 2006) in the assembled genome sequence or the raw genomic sequencing traces.

To determine the structure of the $T$. spiralis SL RNA we mapped the $3^{\prime}$ end of the RNAs derived from $t s l r-1$ and tslr-14 using an approach involving in vitro polyadenylation of T. spiralis total RNA with yeast poly(A) polymerase. This enzyme adds non-genome-encoded adenosine residues to the 3 ' ends of RNA molecules. RNA treated in this way was then reverse transcribed using an oligo dT-anchor primer. We used tslr-1- and tslr-14-specific primers together with the anchor primer to PCR amplify fragments of the introns of $t s l r-1$ and $t s l r-14$, which were subsequently cloned and sequenced (for each gene we sequenced four cDNAs). By comparing the resultant cDNA sequences to genomic sequence and noting at which point nontemplate adenosine residues were added, we were able to map the $3^{\prime}$ ends of the SL RNAs (Fig. 2B). Assuming that there are no nucleotides upstream of the spliced leader, the mapping of the 3' ends of TSL1 gives a size of between 97 and 99 nt for the precursor TSL RNA, very close to that of the canonical nematode SL1 RNA. We noted that the $3^{\prime}$ ends of both RNAs mapped to U-rich sequences, which in several organisms have been associated with transcription termination (Maroney et al. 1990a; Huang and Maraia 2001). We could subsequently identify T-rich sequences toward the $3^{\prime}$ ends of the other $t$ slr genes (Table 2). The structures of the $t s l r-1$ and $t s l r-14$ RNAs were modeled using MFOLD version 2.3 (Zuker 2003), with the only constraint that the Sm-like binding site was required to be single stranded. Both TSL-RNAs adopted the same structure with the splice donor site in a stem-loop and two additional stem-loops flanking the Sm-binding site (Fig. 2B). As was found for other SL RNAs, the putative splice donor site is located in the hairpin formed between the SL and the intron.

Finally, we performed Northern analysis with total RNA from muscle L1 larvae to confirm that $t s l r-1$ and $t s l r-14$ genes are transcribed as small RNAs. As expected each gene-specific anti-sense probe identified an RNA species of $\sim 100$ nt (Fig. 2C) representing the SL-RNA; the smear of larger transcripts detected representing trans-spliced mRNAs. The hybridization signal for $t s l r-1$ was much stronger $(>20$-fold) than that for $t s l r-14$ when we corrected for the specific activity of the probe and exposure times. Most likely this is because $t s l r-14$ is expressed at lower levels, but it may also indicate that it is less efficiently used in trans-splicing events, at least in the muscle L1 larva, than tslr-1. In fact, to date we have not identified any cDNA or EST which utilizes TSL-14 (Fig. 1A).

\section{DISCUSSION}

Recent work from several groups, based upon small-subunit rDNA sequences, has demonstrated that the lineage leading to $T$. spiralis (and the other members of the subclass Dorylaimia) branches close to the base of the nematode phylogenetic tree (Aleshin et al. 1998; Blaxter et al. 1998; Holterman et al. 2006). All other nematodes for which data on trans-splicing is available group together in a single subclass termed Rhabditida (Fig. 3). Our data thus indicate that SL trans-splicing is likely to be an ancestral nematode feature, since it is found in two subclasses whose last common ancestor lies at the origin of the nematode phylum. However, the absence of spliced leaders that are related to the "universal" SL1 found in all other nematodes studied raises questions about whether SL1 and SL1-like spliced leaders are an ancestral nematode feature, lost in the lineage leading to T. spiralis, or whether the diverse SL RNA complement found in T. spiralis is the ancestral state, from which SL1-like spliced leaders evolved. Recently we found that SL trans-splicing also occurs in another Trichinella species, T. pseudospiralis, and that at least some of the TSLs are conserved between species (B. Connolly, unpubl.). An important area for future work will be determining the 


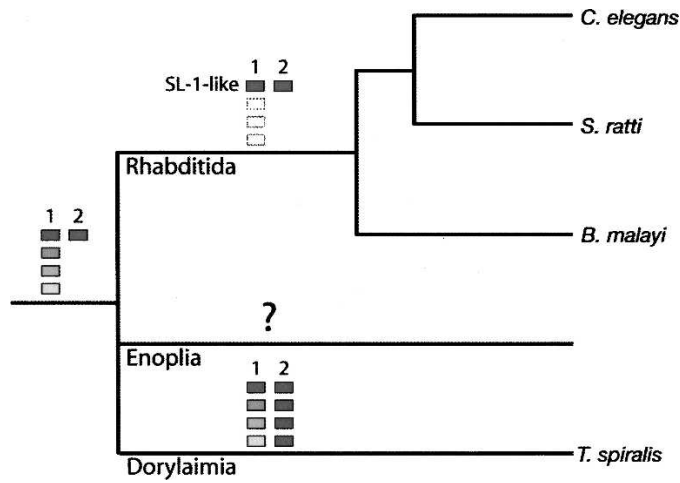

FIGURE 3. Nematode phylogeny showing two possible scenarios to explain extant spliced leader complements. The phylogeny shown is based upon SSU rDNA-based studies (Aleshin et al. 1998; Blaxter et al. 1998; Holterman et al. 2006). All recent studies are agreed on the relative position of $C$. elegans with respect to $T$. spiralis, though the exact topologies differ. Boxes indicate SL RNA genes, with the shading used to indicate distinct genes. In model 1 , the ancestor of all extant nematodes possessed multiple, different SL RNA genes, which were maintained in the lineage leading to T. spiralis. In this model, all but one of these were lost in Rhabditida lineage, the surviving one becoming the ancestor of the SL1 and SL2 RNA genes. In model 2, the ancestral nematode possessed only one (possibly multicopy) SL1like gene, that in the Dorylaimia lineage underwent multiple rounds of duplication and diversification, but was maintained in the Rhabditida lineage. Information on trans-splicing in Enoplid nematodes should shed light on which of these models is correct.

distribution of spliced leaders in other members of the Dorylaimia, as well as in the other major nematode subclass, the Enoplia.

The most striking feature of the family of SL RNA genes that we have identified in T. spiralis is their diversity. Although other nematodes have families of SL RNA genes, these show limited diversity. The T. spiralis spliced leaders show variability at almost all positions, with the exception of the last three residues, and share none of the diagnostic features of SL1-like or SL2-like spliced leaders identified by Guiliano and Blaxter (2006).

We have not been able to show that there are functional differences between the SL RNA genes, although the existence of polymorphic TSLs raises the prospect that each may confer unique translational efficiencies on the recipient mRNA. Beyond this, it was nevertheless clear in our study that some SL RNAs are more frequently used than others. Although we observe a bias in the use of certain TSLs, it is unclear whether this reflects real differences in the efficiency with which each TSL participates in trans-splicing reactions. Furthermore, it remains to be determined whether a bias in TSL utilization persists throughout all life-cycle stages or whether particular TSLs exhibit stage-specific or tissue-specific usage. The preponderance of blgs-1 mRNAs receiving TSL1 contrasts with the observation that GM2 activator protein mRNAs more frequently are trans-spliced to TSL11. If this is due to tissue-specific expression, then one would expect, for instance, that TSL1 would be more strongly expressed in tissues that express blgs-1, compared to other TSL genes. No such tissue-specific expression has been reported for SL RNAs in other nematodes. Furthermore, we recently found that trans-splicing onto the T. pseudospiralis GM2-AP mRNAs also exhibits a strong bias, with TSL7 being trans-spliced onto $10 / 11$ of the 5 'RACE clones examined (B. Connolly, unpubl.).

In a survey of T. spiralis ESTs, only about $1 \%$ of mRNAs were identified with a spliced leader. However, without knowing how many of the ESTs are derived from fulllength transcripts, it is unclear if this low number results from most ESTs being $5^{\prime}$ truncated, and thus fail to include the spliced leader, or whether this actually represents the true proportion of trans-spliced mRNAs. Additionally, given that the T. spiralis genome sequence is incomplete, it remains possible that we have not identified all of the TSLs. Thus, our data may underestimate both the number of TSLs and the proportion of mRNAs that are trans-spliced.

The majority of what we know about the mechanism of trans-splicing in nematodes has largely come from studies in just two nematodes: C. elegans and Ascaris spp. Although not closely related, these nematodes both add the canonical SL1 spliced leader to the majority of their mRNAs (Maroney et al. 1995). Biochemical and molecular genetic investigations have built up a detailed picture of the mechanism and biological significance of spliced leader addition to mRNA in nematodes (Bruzik et al. 1988; Thomas et al. 1988; Maroney et al. 1990a,b; Van Doren and Hirsh 1990; Denker et al. 1996, 2002; Ferguson et al. 1996; Ferguson and Rothman 1999; Lall et al. 2004; Cheng et al. 2007; MacMorris et al. 2007). The conservation of the SL1 primary sequence between C. elegans and A. suum suggests a functional constraint. In addition, all previously identified nematode SLs are clearly variants of SL1. This is in contrast to comparisons between nematode SLs and those from organisms from other phyla, which show no evidence of conserved primary sequence. It is known that one reason for this constraint is in part because the SL1 sequence encodes a transcriptional control element. However, it may also be that SL1 and SL1-like RNAs are constrained by the RNA-protein interactions involved in the formation of the snRNP complexes that form during trans-splicing. In both Ascaris and C. elegans, SL1 snRNPs contain a conserved pair of proteins (Denker et al. 2002; MacMorris et al. 2007). Database searches show that these proteins are also present in Brugia malayi. We have not been able to identify genes encoding homologs of these proteins in the $T$. spiralis genome sequence assembly, suggesting the possibility that they have coevolved with SL1-type spliced leaders. An important question for future studies to address will be the extent to which the mechanisms of trans-splicing differ between C. elegans and T. spiralis.

Operons appear to have coevolved with spliced leader transsplicing as they provide a means for resolving polycistronic 
pre-mRNAs (Blumenthal 2005). The choice of spliced leader that is added to downstream mRNAs processed from such polycistronic RNAs is variable, with some nematodes using the universal SL1, while others have evolved specialized variants apparently derived from SL1 (Guiliano and Blaxter 2006). However, as stated above, the fact that all such nematodes share a common ancestor does not allow any inferences to be made about the use of operons in more distantly related nematodes. None of the genes that we have shown to be trans-spliced in T. spiralis appear to be part of operons, and there is no evidence to suggest that they exist in this nematode. However, the analysis of the $T$. spiralis genome is at an early stage and further work will be required to address the existence of operons in this nematode. The findings described here nevertheless provide an important and revealing insight into the evolution and complexity of mRNA processing in a wide range of taxa.

\section{MATERIALS AND METHODS}

\section{Nematode isolation and RNA preparation}

Trichinella spiralis parasites were maintained in female ICR mice and muscle L1 larvae recovered as previously described (Connolly et al. 1995). Total RNA from T. spiralis muscle L1 larvae was prepared as described previously (Kuratli et al. 1999).

\section{Northern analysis}

Ten micrograms of total RNA were fractionated by $2 \%$ glyoxal/ DMSO agarose gel electrophoresis and transferred to Hybond $\mathrm{N}$ membranes using standard techniques. TSL-1 and TSL-14 RNAs were detected using the ${ }^{32} \mathrm{P}$ labeled oligonucleotide probes antiTSL-1 and anti-TSL-14 (Supplemental Table 3), respectively. Probes are complementary to the respective splice leader sequences and were ${ }^{32} \mathrm{P}$ end-labeled using $\gamma-{ }^{32} \mathrm{P}$ ATP and T4 polynucleotide kinase. Hybridization was performed according to a standard protocol (Church and Gilbert 1984) except that hybridization was at $42^{\circ} \mathrm{C}$ and wash steps were at room temperature, $36^{\circ} \mathrm{C}$, and $42^{\circ} \mathrm{C}$. Blots were analyzed by autoradiography or using a Fuji FLA3000 Phosphorimager and Aida 2.0 software (Raytest GmbH).

\section{Determination of cDNA $5^{\prime}$ ends}

The $5^{\prime}$ ends of cDNAs representing full-length mRNAs were obtained through 5'RACE using the GeneRacer kit (Invitrogen), according to the manufacturer's instructions. Gene-specific primers used are given in the Supplemental Table 3, and the cDNAs, amplified by PCR using the Expand High Fidelity polymerase (Roche), were cloned into pGEM T-Easy (Promega) or pCR4TOPO (Invitrogen) and the resulting plasmid inserts sequenced by the University of Dundee Sequencing Service.

\section{Identification of trans-spliced $T$. spiralis ESTs}

Using each of the TSL sequences we carried out a megaBLAST search of the publicly available T. spiralis EST sequences, looking for matches only at or within $13 \mathrm{nt}$ of the $5^{\prime}$ end of the sequence. We noted that the 12-nt sequence $5^{\prime}$-CCCACGCGTCCG-3' was found at the $5^{\prime}$ end of virtually all ESTs with accession numbers of the form ESxxxx. Independent analysis 5'RACE clones of several of the genes (both trans-spliced and non-trans-spliced) represented by these ESTs failed to identify this sequence at the $5^{\prime}$ ends of the mRNAs (data not shown). We concluded that this sequence is an artifact and we thus excluded it when screening the $5^{\prime}$ end sequence of these ESTs. A BLAST search using the positively identified cDNA sequences was then carried out against the $T$. spiralis-1.0 supercontig assembly (http://genome.wustl.edu/tools/ blast/) to confirm the presence of an outron at the point at which the TSL was predicted to be added.

\section{Identification of predicted TSL RNA genes}

The T. spiralis genome draft release comprising 6261 contigs (downloaded March 23, 2006, from the University of Washington Genome Sequencing Center; ftp://genome.wustl.edu/pub/organism/ invertebrate/Trichinella_spiralis) was surveyed for the presence of novel splice leader genes using a motif search algorithm (Trichinella-SL-scan.pl) written in the language Perl (Active State Perl version 5.005_3). The algorithm employs a sliding search window to look initially within each contig sequence for examples of the Sm-binding motif-like sequence (denoted element 1), with the sequence $5^{\prime}$-AATTTTTGTCCTGGG-3'. If such a sequence is found, the algorithm then searches in a region between 45 and 22 nt upstream ( $\left.5^{\prime}\right)$ of the Sm-binding motif sequence for the splice donor sequence $5^{\prime}$-AAGGTA-3' (element 2). Having found this second element, the algorithm then searches the sequence between elements 1 and 2 for a third element, comprising any sequence capable of forming a stem-loop with stem length of at least $11 \mathrm{nt}$. The algorithm allows G-U wobble base pairing within the stem. During scanning, when an element 1 example is found at a given position, but there is no element 2 within the defined upstream window, the sliding window search automatically resumes for the next 3 ' example of element 1 . The same process is triggered if juxtaposed elements 1 and 2 are found, but no intervening element 3 . The algorithm carries out the search pattern on both DNA strands. The algorithm can operate at varying levels of stringency. Based upon known examples of TSL sequences identified at the $5^{\prime}$ ends of T. spiralis mRNA sequences by this study, the search was carried out allowing three mismatches among $15 \mathrm{nt}$ within the Sm-binding motif, and with three nucleotide mismatches within the first $11 \mathrm{nt}$ of the element 3 stem permitted. Subsequently, identified sequences were manually examined, and only those with a more stringent match to the putative Sm-binding site (one mismatch allowed in $5^{\prime}$-AATTTTTGT-3') were retained as putative $t s l r$ genes. The Perl script is freely available on request from the authors.

\section{TSL RNA 3' end mapping}

TSL RNA 3' ends were determined essentially as described previously (Keall et al. 2007). T. spiralis total RNA (4 $\mu \mathrm{g})$ was poly(A) tailed using yeast poly(A) polymerase, reverse transcribed using an oligo-dT-anchor primer, and then PCR amplified using TSL-specific primers and an anchor primer. PCR fragments were inserted into pGEM-T easy (Promega), and inserts were sequenced at the University of Dundee DNA Sequencing Facility. 
The TSL-1-specific primer was SL1-F1 and the TSL-14-specific primers were SL14-F1 and SL14-F2 (Supplemental Table 3).

\section{Secondary structure predictions}

Secondary structure predictions of the TSL RNAs were performed using MFOLD Version 2.3 (Zuker 2003) using the default folding conditions $\left(1 \mathrm{M} \mathrm{NaCl}, 37^{\circ} \mathrm{C}\right)$, and with the constraint that the Sm-binding site ( $5^{\prime}$-AAUUUUUGU-3') was required to be single stranded.

\section{Accession numbers}

Primary sequence data reported in this article are available in the GenBank database under the accession numbers EF681123 (tslr-1), EF681124 (tslr-14), EF681125 (tll-1), and EF682904 (blgs-1).

\section{SUPPLEMENTAL DATA}

Supplemental materials are available at http://www.abdn.ac. uk/ bch182/PettittSupplTables.pdf.

\section{ACKNOWLEDGMENTS}

The authors thank Diane Massie and Aileen Flett for technical assistance. This work was funded in part by a Wellcome Trust grant to J.P. and B.M. (WT grant 076220).

Received December 7, 2007; accepted December 19, 2007.

\section{REFERENCES}

Aleshin, V.V., Kedrova, O.S., Milyutina, I.A., Vladenskaya, N.S., and Petrov, N.B. 1998. Relationships among nematodes based on the analysis of $18 \mathrm{~S}$ rRNA gene sequences: Molecular evidence for monophyly of chromadorian and secernentian nematodes. Russ. J. Nematol. 6: 175-184.

Blaxter, M.L., De Ley, P., Garey, J.R., Liu, L.X., Scheldeman, P., Vierstraete, A., Vanfleteren, J.R., Mackey, L.Y., Dorris, M., Frisse, L.M., et al. 1998. A molecular evolutionary framework for the phylum Nematoda. Nature 392: 71-75.

Blumenthal, T. 2005. Trans-splicing and operon. In WormBook (ed. The C. elegans Research Community, WormBook), doi: 10.1895/ wormbook.1.7.1., http://www.wormbook.org.

Bruzik, J.P., Van Doren, K., Hirsh, D., and Steitz, J.A. 1988. Trans splicing involves a novel form of small nuclear ribonucleoprotein particles. Nature 335: 559-562.

Cheng, G., Cohen, L., Mikhli, C., Jankowska-Anyszka, M., Stepinski, J., Darzynkiewicz, E., and Davis, R.E. 2007. In vivo translation and stability of trans-spliced mRNAs in nematode embryos. Mol. Biochem. Parasitol. 153: 95-106.

Church, G.M. and Gilbert, W. 1984. Genomic sequencing. Proc. Natl. Acad. Sci. 81: 1991-1995.

Connolly, B., Ingram, L.J., and Smith, D.F. 1995. Trichinella spiralis: Cloning and characterization of two repetitive DNA sequences. Exp. Parasitol. 80: 488-498.

Conrad, R., Thomas, J., Spieth, J., and Blumenthal, T. 1991. Insertion of part of an intron into the $5^{\prime}$ untranslated region of a Caenorhabditis elegans gene converts it into a trans-spliced gene. Mol. Cell. Biol. 11: 1921-1926.

Davis, R.E. 1997. Surprising diversity and distribution of spliced leader RNAs in flatworms. Mol. Biochem. Parasitol. 87: 29-48.

Denker, J.A., Maroney, P.A., Yu, Y.T., Kanost, R.A., and Nilsen, T.W. 1996. Multiple requirements for nematode spliced leader RNP function in trans-splicing. RNA 2: 746-755.
Denker, J.A., Zuckerman, D.M., Maroney, P.A., and Nilsen, T.W. 2002. New components of the spliced leader RNP required for nematode trans-splicing. Nature 417: 667-670.

Ferguson, K.C. and Rothman, J.H. 1999. Alterations in the conserved SL1 trans-spliced leader of Caenorhabditis elegans demonstrate flexibility in length and sequence requirements in vivo. Mol. Cell. Biol. 19: 1892-1900.

Ferguson, K.C., Heid, P.J., and Rothman, J.H. 1996. The SL1 transspliced leader RNA performs an essential embryonic function in Caenorhabditis elegans that can also be supplied by SL2 RNA. Genes \& Dev. 10: 1543-1556.

Ganot, P., Kallesoe, T., Reinhardt, R., Chourrout, D., and Thompson, E.M. 2004. Spliced-leader RNA trans splicing in a chordate, Oikopleura dioica, with a compact genome. Mol. Cell. Biol. 24: 7795-7805.

Guiliano, D.B. and Blaxter, M.L. 2006. Operon conservation and the evolution of trans-splicing in the phylum Nematoda. PLoS Genet. 2: e198. doi: 10.1371/journal.pgen.0020198.

Hastings, K.E. 2005. SL trans-splicing: Easy come or easy go? Trends Genet. 21: 240-247.

Holterman, M., van der Wurff, A., van den Elsen, S., van Megen, H., Bongers, T., Holovachov, O., Bakker, J., and Helder, J. 2006. Phylum-wide analysis of SSU rDNA reveals deep phylogenetic relationships among nematodes and accelerated evolution toward crown Clades. Mol. Biol. Evol. 23: 1792-1800.

Huang, Y. and Maraia, R.J. 2001. Comparison of the RNA polymerase III transcription machinery in Schizosacchromyces pombe, Sacchromyces cerevisiae, and human. Nucleic Acids Res. 29: 2675-2690. doi: 10.1093/nar/29.13.2675.

Keall, R., Whitelaw, S., Pettitt, J., and Muller, B. 2007. Histone gene expression and histone mRNA $3^{\prime}$ end structure in Caenorhabditis elegans.. BMC Mol. Biol. 8: 51.

Krause, M. and Hirsh, D. 1987. A trans-spliced leader sequence on actin mRNA in C. elegans. Cell 49: 753-761.

Kuratli, S., Lindh, J.G., Gottstein, B., Smith, D.F., and Connolly, B. 1999. Trichinella spp.: Differential expression of two genes in the muscle larva of encapsulating and nonencapsulating species. Exp. Parasitol. 93: 153-159.

Lall, S., Friedman, C.C., Jankowska-Anyszka, M., Stepinski, J., Darzynkiewicz, E., and Davis, R.E. 2004. Contribution of transsplicing, 5'-leader length, cap-poly(A) synergism, and initiation factors to nematode translation in an Ascaris suum embryo cellfree system. J. Biol. Chem. 279: 45573-45585.

Lindh, J.G., Connolly, B., McGhie, D.L., and Smith, D.F. 1998. Identification of a developmentally regulated Trichinella spiralis protein that inhibits MyoD-specific protein: DNA complexes in vitro. Mol. Biochem. Parasitol. 92: 163-175.

MacMorris, M., Kumar, M., Lasda, E., Larsen, A., Kraemer, B., and Blumenthal, T. 2007. A novel family of C. elegans snRNPs contains proteins associated with trans-splicing. RNA 13: 511-520.

Maroney, P.A., Denker, J.A., Darzynkiewicz, E., Laneve, R., and Nilsen, T.W. 1995. Most mRNAs in the nematode Ascaris lumbricoides are trans-spliced: A role for spliced leader addition in translational efficiency. RNA 1: 714-723.

Maroney, P.A., Hannon, G.J., Denker, J.A., and Nilsen, T.W. 1990a. The nematode spliced leader RNA participates in trans-splicing as an Sm snRNP. EMBO J. 9: 3667-3673.

Maroney, P.A., Hannon, G.J., and Nilsen, T.W. 1990b. Transcription and cap trimethylation of a nematode spliced leader RNA in a cellfree system. Proc. Natl. Acad. Sci. 87: 709-713.

Mattaj, I.W. 1988. U snRNP assembly and transport. In Structure and function of major and minor smal nuclear ribonucleoprotein particles (ed. M. L. Birnstiel), pp. 100-114. Springer Verlag, New York.

Nilsen, T.W. 2001. Evolutionary origin of SL-addition trans-splicing: Still an enigma. Trends Genet. 17: 678-680.

Pouchkina-Stantcheva, N.N. and Tunnacliffe, A. 2005. Spliced leader RNA-mediated trans-splicing in phylum Rotifera. Mol. Biol. Evol. 22: $1482-1489$. 
Satou, Y., Hamaguchi, M., Takeuchi, K., Hastings, K.E., and Satoh, N. 2006. Genomic overview of mRNA 5 '-leader trans-splicing in the ascidian Ciona intestinalis. Nucleic Acids Res. 34: 3378-3388.

Spieth, J., Brooke, G., Kuersten, S., Lea, K., and Blumenthal, T. 1993. Operons in C. elegans: Polycistronic mRNA precursors are processed by trans-splicing of SL2 to downstream coding regions. Cell 73: 521-532.

Stover, N.A. and Steele, R.E. 2001. Trans-spliced leader addition to mRNAs in a cnidarian. Proc. Natl. Acad. Sci. 98: 5693-5698.

Sutton, R.E. and Boothroyd, J.C. 1986. Evidence for trans splicing in trypanosomes. Cell 47: 527-535.

Tessier, L.H., Keller, M., Chan, R.L., Fournier, R., Weil, J.H., and Imbault, P. 1991. Short leader sequences may be transferred from small RNAs to pre-mature mRNAs by trans-splicing in Euglena. EMBO J. 10: 2621-2625.

Thomas, J.D., Conrad, R.C., and Blumenthal, T. 1988. The C. elegans trans-spliced leader RNA is bound to Sm and has a trimethylguanosine cap. Cell 54: 533-539.
Thomas, J., Lea, K., Zucker-Aprison, E., and Blumenthal, T. 1990. The spliceosomal snRNAs of Caenorhabditis elegans. Nucleic Acids Res. 18: 2633-2642. doi: 10.1093/nar/18.9.2633.

Van Doren, K. and Hirsh, D. 1990. mRNAs that mature through trans-splicing in Caenorhabditis elegans have a trimethylguanosine cap at their 5' termini. Mol. Cell. Biol. 10: 1769-1772.

Vandenberghe, A.E., Meedel, T.H., and Hastings, K.E. 2001. mRNA 5'-leader trans-splicing in the chordates. Genes \& Dev. 15: 294-303.

Zayas, R.M., Bold, T.D., and Newmark, P.A. 2005. Spliced-leader trans-splicing in freshwater planarians. Mol. Biol. Evol. 22: 20482054.

Zhang, H., Hou, Y., Miranda, L., Campbell, D.A., Sturm, N.R., Gaasterland, T., and Lin, S. 2007. Spliced leader RNA transsplicing in dinoflagellates. Proc. Natl. Acad. Sci. 104: 4618-4623.

Zuker, M. 2003. Mfold web server for nucleic acid folding and hybridization prediction. Nucleic Acids Res. 31: 3406-3415. doi: 10.1093/nar/gkg595. 

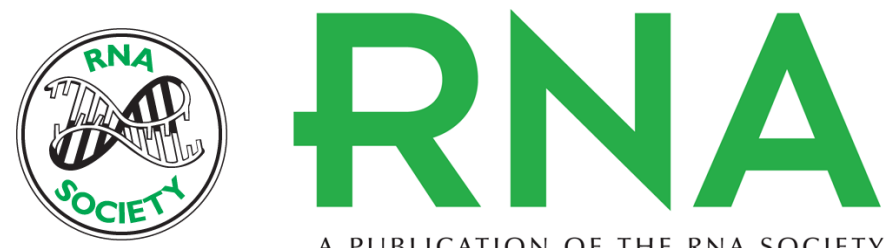

A PUBLICATION OF THE RNA SOCIETY

\section{Spliced leader trans-splicing in the nematode Trichinella spiralis uses highly polymorphic, noncanonical spliced leaders}

Jonathan Pettitt, Berndt Müller, Ian Stansfield, et al.

RNA 2008 14: 760-770

References This article cites 40 articles, 14 of which can be accessed free at:

http://rnajournal.cshlp.org/content/14/4/760.full.html\#ref-list-1

License

Email Alerting Receive free email alerts when new articles cite this article - sign up in the box at the Service top right corner of the article or click here. 